\title{
Echocardiographic demonstration of aortic root abscess after infective endocarditis
}

\author{
C M WONG, P OLDERSHAW, D G GIBSON \\ From the Cardiac Department, Brompton Hospital, London
}

SUMmARY We describe a patient with infective endocarditis in whom the diagnosis was apparent only on two-dimensional echocardiography. There was no clinical or haematological evidence of active endocarditis and the diagnosis was made by the demonstration of an abscess cavity and vegetation posterior and lateral to the aortic root, deforming the left atrial cavity. The findings were confirmed by cardiac catheterisation and operation.

Aortic regurgitation is a well-known complication of infective endocarditis involving the aortic valve. The presentation may be acute or even life threatening within the first few days. We present a patient with aortic regurgitation of insidious onset, in whom active endocarditis was not apparent on clinical grounds, and in whom the diagnosis was made echocardiographically and confirmed by angiography and operative findings.

\section{Case report}

A 32-year-old woman with a previous history of rheumatic fever developed infective endocarditis in June 1979 during the late stages of pregnancy. The diagnosis was confirmed on blood cultures, the organism being Streptococcus viridans. She was treated with a six-week course of benzylpenicillin in adequate dosage, and was discharged symptom free with a normal haemoglobin, white blood cell count, erythrocyte sedimentation rate, and sterile blood cultures. She remained well over the next six months but then developed gradual onset of increasing exertional dyspnoea, orthopnoea, and paroxysmal nocturnal dyspnoea. These symptoms progressed over two months and she was admitted to hospital. On examination she was apyrexial with no systemic manifestations of endocarditis. In the cardiovascular system she was in sinus rhythm, blood pressure was $170 / 40 \mathrm{mmHg}$, with a rapid upstroke pulse. The apical impulse was sustained. There were normal first and second sounds with a grade $3 / 4$ early diastolic murmur at the left sternal edge. A grade 2/4 mid-diastolic murmur (Austin Flint) was also heard at the apex. The rest of the examination was normal.

\section{INVESTIGATIONS}

Haemoglobin $14.7 \mathrm{~g} / 100 \mathrm{ml}$, white blood cells $7300 /$ $\mathrm{mm}^{3}$, erythrocyte sedimentation rate $3 \mathrm{~mm} / \mathrm{h}$. Urea and electrolytes were normal. Liver function tests were normal. Three blood cultures were sterile. There was no haematuria on urine microscopy. Antifungal antibodies were not raised. Electrocardiogram showed sinus rhythm, PR interval $0.24 \mathrm{~s}$, normal QRS axis, left ventricular hypertrophy on voltage criteria, ST segment depression anterolaterally. Chest $x$-ray film showed cardiac enlargement (CTR 17/30), with upper lobe venous distension.

\section{ECHOCARDIOGRAPHIC FINDINGS}

M-mode echocardiogram showed normal aortic root, with premature opening of aortic valve leaflets (Fig. 1a), left atrial dimension was normal, and the mitral valve leaflets were normal. The left ventricle was enlarged at $7 \mathrm{~cm}$ in end-diastole and $5 \mathrm{~cm}$ in end-systole and peak rate of dimension increase raised to $17 \mathrm{~cm} / \mathrm{s}$, with the rapid filling phase prolonged to $0.21 \mathrm{~s}$. These findings are compatible with severe aortic regurgitation. ${ }^{1}$ Two-dimensional echocardiography showed normal aortic root and valve in the long axis view, but in both the long axis view, with the transducer directed a little leftward, and in the short axis view, a diverticulum was seen posterior and to the left of the aortic root arising from the sinus of Valsalva and bulging into but not connecting with the left atrium. An abnormal and mobile echo mass was seen 
Fig. 1 (a) M-mode echocardiogram of aortic root. ECG, electrocardiogram; $A A W$, anterior aortic wall; $P A W$, posterior aortic wall; $A V$, aortic valve; $L A$, left atrium; $A V O$, aortic valve opening. (b) M-mode echo of left ventricular cavity. ECG, electrocardiogram; PCG, phonocardiogram; IVS, interventricular septum; $M V$ mitral valve; $A C G$, apexcardiogram; $E N$, endocardium; EP, epicardium.

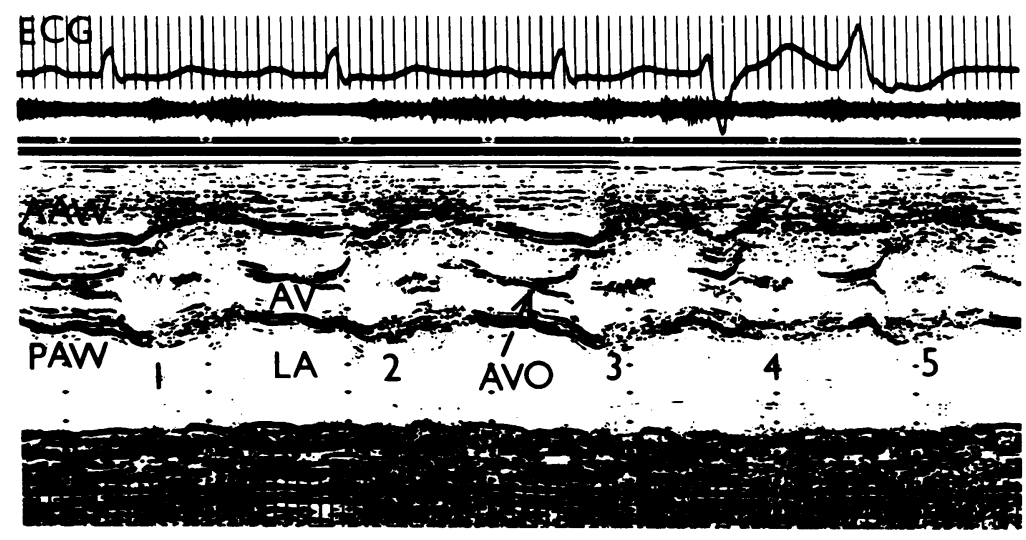

(a)
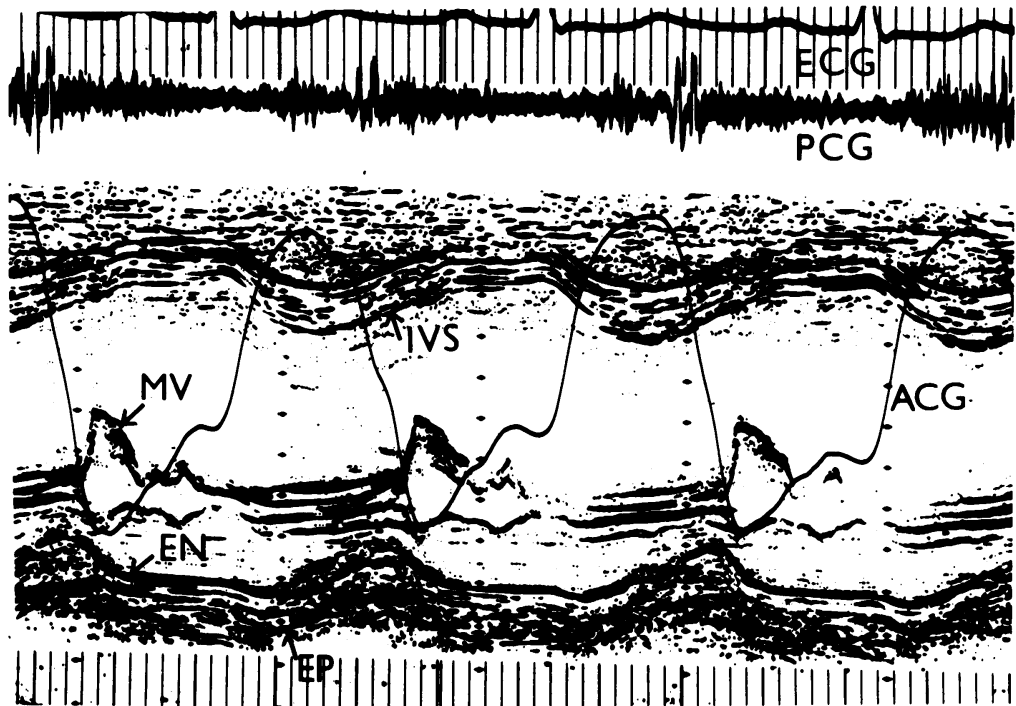

(b)

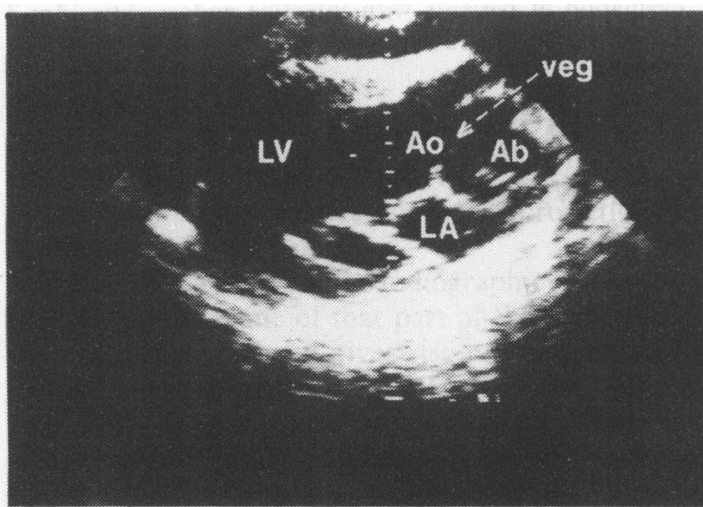

(a)

Fig. 2 (a) $2 D$ echo, "off axis" long axis view. $L V$, left ventricle; $L A$, left atrium; $A b$, abscess cavity; Ao, aortic root; veg, vegetation. (b) $2 D$ echo, short axis view. Ao aortic root; veg, vegetation; LA, left atrium; $A b$, abscess cavity. 
in the mouth of the diverticulum protruding into the sinus of Valsalva (Fig. 2a \& 2b).

\section{CARDIAC CATHETERISATION}

At cardiac catheterisation, the ascending aortic pressure was $140 / 50 \mathrm{mmHg}$; the left ventricular end-diastolic pressure was $40 \mathrm{mmHg}$. Aortogram confirmed severe aortic regurgitation and the presence of a diverticulum arising from the posterior segment of the sinus of Valsalva. Contrast remained in the diverticulum and did not pass into the left atrium.

\section{OPERATION}

The aortic valve was tricuspid, with perforations of the right and non-coronary leaflets. The left coronary leaflet was almost completely detached except at its commissures; there were vegetations on the detached margins. There was a large cavity extending from the posterior sinus of Valsalva towards the left atrium. This cavity was well endothelialised, with no active ulceration or connection with any other chamber of the heart. The aortic valve was completely excised and replaced with a $25 \mathrm{~mm}$ Björk-Shiley prosthesis. The excised valve grew Streptococcus viridans and the patient was treated with a further course of benzylpenicillin.

\section{Discussion}

The use of an M-mode echocardiogram in aortic regurgitation is well established. ${ }^{2}$ Not only is it useful in assessing the severity of aortic regurgitation, the presence or absence of coexisting mitral valve disease, and left ventricular dysfunction, but the aetiology of aortic regurgitation is often identified, for example aortic valve disease, aortic dissection, infective endocarditis, aortic valve prolapse, or rupture of the sinus of Valsalva. Two-dimensional echocardiography has a greater sensitivity and specificity ${ }^{3}$ in establishing the aetiology of aortic regurgitation, and this is well illustrated in this case. The patient presented with severe aortic regurgitation of gradual onset. There was no evidence of persistence of infective endocarditis either at the time of discharge in July 1979 or on subsequent admission. Blood tests, including haemoglobin, white blood cell counts, and erythrocyte sedimentation rate were within normal limits, and blood cultures were all negative. M-mode echocardiography showed signs of severe aortic regurgitation with an enlarged left ventricle, an increased diastolic filling rate, and premature aortic valve opening.

The possibility of persisting infective endocarditis was raised by the echocardiographic demonstration of a diverticulum adjacent to the aortic root. Though this might have been the result of a sinus of Valsalva aneurysm, the presence of a vegetation strongly suggested infective endocarditis as the most likely aetiology. Both $M$-mode and standard 2D long axis views failed to show any abnormality behind the aortic root reflecting the posterolateral position of the abscess cavity.

The presence of such an aortic abscess is likely to increase the risks of aortic valve surgery; thus its preoperative detection and localisation are of surgical significance. Two-dimensional echocardiography using multiple standard and non-standard views should thus be performed on any patient undergoing surgery for aortic regurgitation after infective endocarditis.

\section{References}

1 Upton MT, Gibson DG. The study of left ventricular function from digitized echocardiograms. Progr Cardiovasc Dis 1978; 20: 359-84.

2 Feigenbaum H. Echocardiography. 2nd ed. New York: Lea \& Febiger, 1976.

3 Mardelli TJ, Ogawa S, Hubbard FE, Dreifus LS, Meixell LL. Cross-sectional echocardiographic detection of aortic ring abscess in bacterial endocarditis. Chest 1978; 74: 576-8.

4 Mintz GS, Kotler MN, Segal BL, Parry WR. Comparison of two-dimensional and M-mode echocardiography in the evaluation of patients with infective endocarditis. $A m \mathcal{F}$ Cardiol 1979; 43: 738-44.

Requests for reprints to Dr D G Gibson, Cardiac Department, Brompton Hospital, Fulham Road, London SW3 6HP. 